\title{
Experimental clathrate superhydrides $\mathrm{EuH}_{6}$ and $\mathrm{EuH}_{9}$ at extreme pressure conditions
}

\author{
Liang Ma, ${ }^{1,2,3,{ }^{*}}$ Mi Zhou,,${ }^{2}{ }^{*}$ Yingying Wang, ${ }^{1,2}$ Saori Kawaguchi, ${ }^{4}$ Yasuo Ohishi, ${ }^{4}$ Feng Peng, ${ }^{5}$ Hanyu Liu $\odot,{ }^{2}$ \\ Guangtao Liu, ${ }^{2, \dagger}$ Hongbo Wang $\odot,{ }^{1,2, \sharp}$ and Yanming Ma $\oplus^{1,2,3,8}$ \\ ${ }^{1}$ State Key Laboratory of Superhard Materials, College of Physics, Jilin University, Changchun 130012, China \\ ${ }^{2}$ International Center of Computational Method \& Software, College of Physics, Jilin University, Changchun 130012, China \\ ${ }^{3}$ International Center of Future Science, Jilin University, Changchun 130012, China \\ ${ }^{4}$ Japan Synchrotron Radiation Research Institute, Sayo, Hyogo 679-5198, Japan \\ ${ }^{5}$ College of Physics and Electronic Information, Luoyang Normal University, Luoyang 471022, China
}

(Received 26 July 2020; accepted 26 October 2021; published 12 November 2021)

\begin{abstract}
The recent discovery of a class of sodalitelike clathrate superhydrides (e.g., $\mathrm{YH}_{6}, \mathrm{YH}_{9}, \mathrm{ThH}_{9}, \mathrm{ThH}_{10}$, and $\left.\mathrm{LaH}_{10}\right)$ at extreme pressures, which commonly exhibit high-temperature superconductivity with the highest $T_{c}$ approaching $260 \mathrm{~K}$ for $\mathrm{LaH}_{10}$, opened up a new era in the search for high-temperature superconductors in metal superhydrides. There is high interest in finding alternative clathrate superhydrides that might witness the long-dreamed room-temperature superconductivity. Here, we target the experimental synthesis of europium (Eu) superhydrides where theory can fail for the prediction of superconductivity. We pressurized and laser heated a mixture of metal $\mathrm{Eu}$ and ammonia borane $\left(\mathrm{NH}_{3} \mathrm{BH}_{3}\right)$ in a diamond-anvil cell and successfully synthesized the clathrate structured $\mathrm{EuH}_{6}$ and $\mathrm{EuH}_{9}$ at conditions of $152 \mathrm{GPa}$ and $1700 \mathrm{~K}$, and $170 \mathrm{GPa}$ and $2800 \mathrm{~K}$, respectively. Two nonclathrate structured phases of $\mathrm{EuH}_{5}$ and $\mathrm{EuH}_{6}$ were also synthesized that are not reported in lanthanide superhydrides. Theoretical simulations predicted that all the synthesized europium hydrides are magnetic, where the electrical resistance measurements suggest a possible magnetic order transition temperature at around 225 and $258 \mathrm{~K}$, respectively, for $\mathrm{EuH}_{5}$ and clathrate $\mathrm{EuH}_{6}$. Our work has created a model superhydride platform for subsequent investigations on how a strongly correlated effect and magnetism can affect the superconductivity of superhydrides.
\end{abstract}

DOI: 10.1103/PhysRevResearch.3.043107

\section{INTRODUCTION}

The quest for atomic metallic hydrogen (AMH) has proven extremely challenging due to the requirements of ultrahigh-pressure conditions and supersensitive characterizations [1-4]. Alternatively, in 2012, there was a theoretical proposal on potential high $T_{c}$ superconductivity in superhydride $\mathrm{CaH}_{6}$ (a theoretical $T_{c}=235 \mathrm{~K}$ at $150 \mathrm{GPa}$ ) stabilized at high pressures [5]. The key to the unusually high $T_{c}$ superconductivity lies in the formation of a Ca-doped $\mathrm{AMH}$ within a peculiar $\mathrm{H}$ clathrate structure containing enclathrated $\mathrm{Ca}$ in a crystal lattice, giving rise to a large $\mathrm{H}$-derived electronic density of states (DOS) at the Fermi level and extremely strong electron-phonon coupling related to $\mathrm{H}-\mathrm{H}$ vibrations in the $\mathrm{H}$ cages [5]. The formation of a clathrate structure in group IV elements is common since the elements' four valence electrons are ready to accept four covalent bonds to stabilize the clathrate cage [6,7]. However, such a clathrate

\footnotetext{
*These authors contributed equally to this work.

${ }^{\dagger}$ liuguangtao@jlu.edu.cn

${ }^{\ddagger}$ whb2477@jlu.edu.cn

$\S$ mym@jlu.edu.cn
}

Published by the American Physical Society under the terms of the Creative Commons Attribution 4.0 International license. Further distribution of this work must maintain attribution to the author(s) and the published article's title, journal citation, and DOI. structure for hydrogen that contains only one valence electron is quite unusual, and becomes possible only when hydrogen accepts a sufficient number of extra electrons from $\mathrm{Ca}$ atoms under high-pressure conditions [5].

Following the first prediction of $\mathrm{CaH}_{6}$, the same clathrate structure was later proposed in $\mathrm{YH}_{6}$ and $\mathrm{MgH}_{6}$ with a predicted $T_{c}=264 \mathrm{~K}$ at $120 \mathrm{GPa}[8]$ and $T_{c}=260 \mathrm{~K}$ at $300 \mathrm{GPa}$ [9], respectively. These two latter superhydrides together with $\mathrm{CaH}_{6}$ share a common feature of high-temperature superconductivity with theoretical $T_{c}$ values all exceeding $200 \mathrm{~K}$. This appearance inevitably generated a great deal of attention towards finding of high $T_{c}$ superconductors in clathrate superhydrides $[10,11]$.

In 2017, along the line of finding clathrate structures in superhydrides, major theoretical progress was achieved via a comprehensive crystal structure searching simulation on rareearth (RE) superhydrides at high pressures [10]. The results elucidated that the pressure-induced formation of clathrate structures is a general behavior for all RE superhydrides. Besides $\mathrm{REH}_{6}$ that shares the same clathrate structure with that of $\mathrm{CaH}_{6}$, two other unexpected clathrate stoichiometries of $\mathrm{REH}_{9}$ and $\mathrm{REH}_{10}$ having a higher $\mathrm{H}$ content than that of $\mathrm{CaH}_{6}$ were also reported. Though all of them are clathrate structures, different $\mathrm{H}_{24}, \mathrm{H}_{29}$, and $\mathrm{H}_{32}$ cages for stoichiometries $\mathrm{REH}_{6}$, $\mathrm{REH}_{9}$ and $\mathrm{REH}_{10}$, respectively, emerged, which could be accepted as a critical role in improving the superconductivity in these superhydrides. Notably, high-temperature superconductivity appears frequently in these RE superhydrides with 
the predicted $T_{c}=276 \mathrm{~K}$ at $150 \mathrm{GPa}$ for $\mathrm{YH}_{9}, T_{c}=303 \mathrm{~K}$ at $400 \mathrm{GPa}$ for $\mathrm{YH}_{10}$, and $T_{c}=288 \mathrm{~K}$ at $200 \mathrm{GPa}$ for $\mathrm{LaH}_{10}$ [10]. It should be particularly emphasized that the predicted clathrate structure and high-temperature superconductivity of $\mathrm{LaH}_{10}$ and $\mathrm{YH}_{10}$ in our work of Ref. [10] coincide exactly with the results from another independent theoretical work [11] appearing at nearly the same time.

With the guidance of the theoretical prediction in Refs. [10,11], experimental progress on the synthesis of these clathrate superhydrides has been remarkable [12-27]. A major move was made on the observation of near-roomtemperature superconductivity in the clathrate $\mathrm{LaH}_{10}$ with $T_{c}$ approaching $260 \mathrm{~K}[12,13]$. Subsequent syntheses of other clathrate superhydrides of $\mathrm{YH}_{6}[19,21], \mathrm{YH}_{9}[20,21], \mathrm{ThH}_{9}$, $\mathrm{ThH}_{10}$ [16], $\mathrm{CeH}_{9}, \mathrm{CeH}_{10}$ [26], (LaY)H $\mathrm{H}_{6},(\mathrm{LaY}) \mathrm{H}_{10}$ [22], and probable $\mathrm{CaH}_{6}[24,25]$ with observed high $T_{c}$ values in the range of 146-253 K added more examples to the family of clathrate superhydrides, a new class of high-temperature superconductors holding a highest $T_{c}$ value of $260 \mathrm{~K}$. The clathrate superhydrides $\mathrm{PrH}_{9}$ and $\mathrm{NdH}_{9}$ that were first predicted in Ref. [10] were also experimentally synthesized $[18,27]$, but the superconducting transition temperatures were measured to be below $10 \mathrm{~K}$, where the superconductivity is apparently suppressed by the emergence of magnetic ordering as suggested. These results indicate, beside hydrogen clathrate cages, other physical properties are also important for affecting the superconductivity of superhydrides, e.g., magnetism and strongly correlated effects. Solving this issue requires advances in exploring the stable structures and fundamental physics properties (magnetism, strongly correlated effects, superconductivity, etc.) of clathrate superhydrides.

$\mathrm{Eu}$, one of the most reactive rare-earth elements and an electronically strongly correlated metal [28,29], adopts a divalent state at an ambient condition with a strong local magnetic moment [30]. At high pressure, magnetic order in $\mathrm{Eu}$ is observed to collapse just above $80 \mathrm{GPa}$ as superconductivity emerges, even though there are strong local $4 f^{7}$ magnetic moments at higher pressure [30-33]. In the H-rich conditions, Eu can accept hydrogen to form dihydrides or even trihydrides below $10 \mathrm{GPa}[34,35]$. Our previous simulations [10] predicted that Eu can react with hydrogen to form the clathrate superhydrides $\mathrm{EuH}_{6}, \mathrm{EuH}_{9}$, and $\mathrm{EuH}_{10}$ at high pressures.

In this paper, we target the synthesis of Eu superhydrides under high-pressure conditions using a laser-heated diamondanvil cell technique via a mixture of Eu and ammonia borane with the aim of finding clathrate superhydrides, allowing us to subsequently investigate how magnetism and a strongly correlated effect in the electronic structure can affect the superconductivity of superhydrides. Encouragingly, we did synthesize a series of Eu superhydrides of $\mathrm{EuH}_{3}, \mathrm{EuH}_{5}, \mathrm{EuH}_{6}$, and $\mathrm{EuH}_{9}$ in the pressure range of 80-170 GPa. All the synthesized europium hydrides are found to be magnetic through our theoretical calculations.

\section{METHODS}

The experiments in the present work were conducted using laser-heated diamond-anvil cell (DAC) techniques. The diamonds used in DACs had a culet with a diameter of 60 $80 \mu \mathrm{m}$ and were beveled at $8^{\circ}$ to a diameter of about $280 \mu \mathrm{m}$. Europium hydrides were synthesized via a reaction of Eu (Alfa Aesar $99.99 \%$ purity) and $\mathrm{BH}_{3} \mathrm{NH}_{3}$ (Sigma-Aldrich 97\% purity) at high-pressure and high-temperature conditions. The use of $\mathrm{BH}_{3} \mathrm{NH}_{3}$ as a source of $\mathrm{H}_{2}$ has been demonstrated to be reliable by previous excellent results $[12,17,19]$. Composite gaskets consisting of a rhenium outer annulus and a mixture of cubic boron nitride ( $c$-BN) and an epoxy (EmbedIt $^{\mathrm{TM}}$ Low Viscosity Epoxy Kit) insert was employed to contain the sample while isolating the electrical leads in the electrical measurements. The temperature dependence of resistance was measured via the four-probe van der Pauw method with four Pt electrodes. Sample preparation and initial loading of the anvils were done in an inert Ar atmosphere (less than 0.01 ppm of oxygen and water) in a glove box to guarantee that the sample was properly isolated from the surrounding atmosphere. Afterwards, the sample was compressed to the target pressure at room temperature. The pressures in all the experiments were determined from the shift in the high-frequency edge of the Raman spectrum gathered from the stressed tip of the diamond anvil at room temperature [36]. In our experience, we found that the pressure increases slightly by about 5 GPa during the cooling process.

In situ $\mathrm{x}$-ray diffraction (XRD) data presented in this work were collected at the BL10XU beamline at the SPring- 8 facility (Hyogo, Japan) [37] with a wavelength of $0.4136 \AA$, and the $\mathrm{x}$-ray spot size was around $3 \mu \mathrm{m} \times 2 \mu \mathrm{m}$. An imaging plate detector (RAXIS-IV; Rigaku) was used to collect the angle-dispersive XRD data. Primary processing and integration of the powder patterns were carried out using the DIOPTAS software [38]. Parts of the preliminary XRD measurements were also performed at the Shanghai Synchrotron Radiation Facility Beamline BL15U1 and Beijing Synchrotron Radiation Facility HP-Station 4W2. The Rietveld refinements were done using GSAS and EXPGUI packages [39]. The laser-heating experiments were performed by a two-sided SPI fiber laser with $1050 \mathrm{~nm}$ at BL10XU in SPring-8, and the temperature was determined by fitting the emission spectra from the surface of the heated sample to Planck's radiation law. All the crystal structure information obtained in this work is summarized in Table S1 [40].

The equations of state (EOS) of $\mathrm{EuH}_{2}, \mathrm{EuH}_{3}, \mathrm{EuH}_{6}$, and $\mathrm{EuH}_{9}$ phases were calculated using density functional theory (DFT) [41,42] within the generalized gradient approximation (Perdew-Burke-Ernzerhof functional) [43], and the projector augmented-wave method $[44,45]$ as implemented in the VASP code [46-48]. The electron-ion interaction was described with the $5 s^{2} 6 s^{2} 5 p^{6} 4 f^{7}$ and $1 s^{1}$ configurations treated as valence electrons for $\mathrm{Eu}$ and $\mathrm{H}$, respectively. To ensure that all enthalpy calculations were well converged to about $1 \mathrm{meV} /$ atom, the Brillouin zone was sampled using $\Gamma$-centered $k$-point meshes with a sufficient density $(2 \pi \times$ $0.03 \AA^{-1}$ ) in reciprocal space, as well as a kinetic energy cutoff of $800 \mathrm{eV}$. Eu has a half-filled $f$ shell, therefore the on-site Coulomb interactions are described by using the DFT $+U$ method with $U=7.0 \mathrm{eV}$ [49]. The dependences of the volume on pressure were fitted by the third-order BirchMurnaghan equation [50] to determine the main parameters of the EOS. 


\section{RESULTS AND DISCUSSION}

In our earlier theoretical study [10], a convex hull of Eu hydrides was constructed through density functional totalenergy calculations to find that $\mathrm{EuH}_{4}$ and clathrate $\mathrm{EuH}_{9}$ are stable compounds, while clathrate $\mathrm{EuH}_{6}$ and $\mathrm{EuH}_{10}$ lie above the convex hull in the whole pressure range of 100-400 GPa. Motivated by the theoretical results, we prepared four DACs referred to as samples $\mathrm{C} 1, \mathrm{C} 2, \mathrm{C} 3$, and $\mathrm{C} 4$, where a $2-\mu \mathrm{m}$ thick sample of Eu was sandwiched between two $\mathrm{BH}_{3} \mathrm{NH}_{3}$ layers in a $c$-BN sample chamber. All samples are pressurized to $80-170 \mathrm{GPa}$ in order to synthesize the clathrate superhydrides.

In sample $\mathrm{C} 1$, the pressure was loaded to $80 \mathrm{GPa}$ at room temperature and then heated to $1400 \mathrm{~K}$ by a laser. The measured XRD pattern was plotted in Fig. 1(a). We found that the resultant products were dominated by an fcc lattice, which could be identified as $\mathrm{EuH}_{2}$ or $\mathrm{EuH}_{3}$. However, it is difficult to distinguish them based on the current experimental data since their difference in lattice volume is too small. We then performed the enthalpy calculation and found that $\mathrm{EuH}_{3}$ has a much lower enthalpy of $0.557 \mathrm{eV} /$ atom than that of $\mathrm{EuH}_{2}+\frac{1}{2} \mathrm{H}_{2}$ when $\mathrm{H}_{2}$ is in an excessive environment. From such an energy consideration, the fcc phase was thus identified as $\mathrm{EuH}_{3}$. It is noteworthy that $\mathrm{EuH}_{3}$ with a cubic close packing of Eu atoms is isostructural to the ambient structure of $\mathrm{LaH}_{3}$ [51], where each $\mathrm{Eu}$ atom is coordinated by eight $\mathrm{H}$ atoms [Fig. 1(b)]. The shortest H-H distance is $2.01 \AA$ at $80 \mathrm{GPa}$.

After the first heating, the sample was further compressed to $130 \mathrm{GPa}$ and then heated to $\sim 1600 \mathrm{~K}$. The integrated XRD pattern is shown in Fig. 1(c). A new phase was evident and considered to be a cubic lattice which is similar to the structure of $\beta-\mathrm{UH}_{3}$ [52]. But from an EOS comparison, we found the experimental volume of the new phase is much larger than the calculated volume of the $\mathrm{EuH}_{3}$ structure (Fig. S1 [40]), therefore, we considered the presence of additional hydrogen in the same metal sublattice with that of $\beta-\mathrm{UH}_{3}$. Structure searches of stoichiometric $\mathrm{EuH}_{4}, \mathrm{EuH}_{5}$, and $\mathrm{EuH}_{6}$ at $150 \mathrm{GPa}$ with a fixed position of Eu atoms were performed using the swarm-intelligence-based CALYPSO structure prediction method [53,54]. We predicted a metastable $P m \overline{3} n$ structure of $\mathrm{EuH}_{5}$ [Fig. 1(d)], the EOS of which is consistent with experimental $P-V$ data (Fig. S1 [40]). This structure contains two inequivalent positions of Eu: One Eu atom in position I is surrounded by $12 \mathrm{H}$ atoms at the corners of an icosahedron and another Eu atom in position II is surrounded by six $\mathrm{H}$ atoms forming a hexahedron. $\mathrm{H}_{2}$ quasimolecules are evenly distributed in the interspace of icosahedrons.

In sample $\mathrm{C} 2$, the initial pressure was loaded up to $92 \mathrm{GPa}$ and heated to a temperature of $3100 \mathrm{~K}$. The XRD patterns [Fig. 1(e)] obtained indicate an unexpected hexagonal structure of $\mathrm{EuH}_{6}$ which is a nonclathrate structure and was first theoretically reported in $\mathrm{ScH}_{6}$ [55], consisting of $\mathrm{H}$-sharing 12-fold $\mathrm{EuH}_{12}$ octahedrons [Fig. 1(f)]. In this structure, hydrogen takes a quasimolecular state with $d(\mathrm{H}-\mathrm{H})=1.143 \AA$ A.

In order to synthesize the targeted clathrate structures, sample C3 was directly compressed to an ultrahigh pressure of $152 \mathrm{GPa}$ and laser heated to a temperature of $1700 \mathrm{~K}$. The measured XRD patterns of the sample [Fig. 2(a)] match the sodalitelike clathrate $\mathrm{EuH}_{6}$ predicted in Ref. [10]. In the (a)

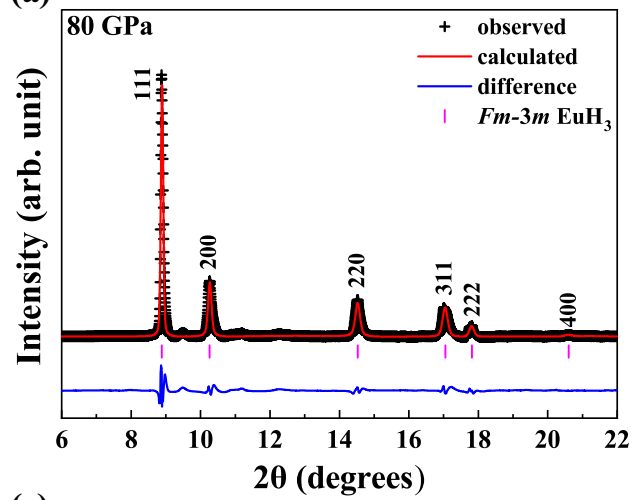

(b) $\mathrm{EuH}_{3}$

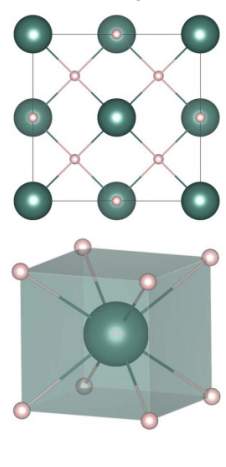

(c)

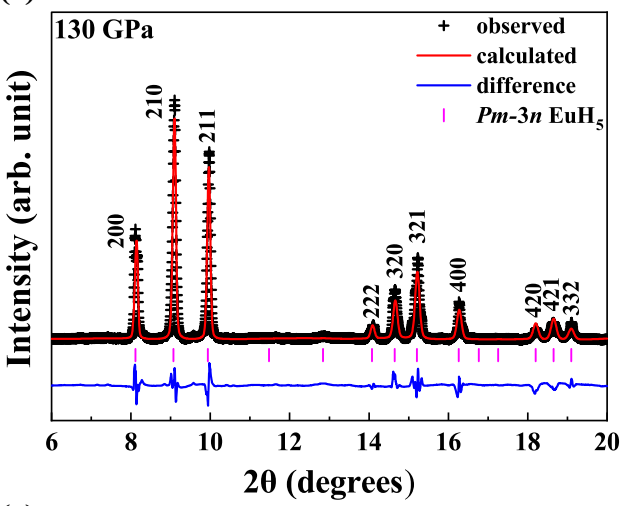

(d) $\mathrm{EuH}_{5}$

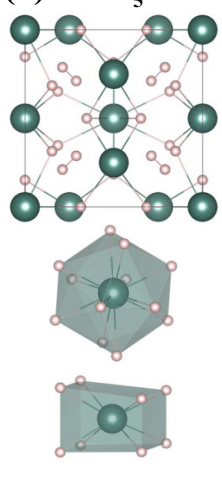

(f) $\mathrm{EuH}_{6}$
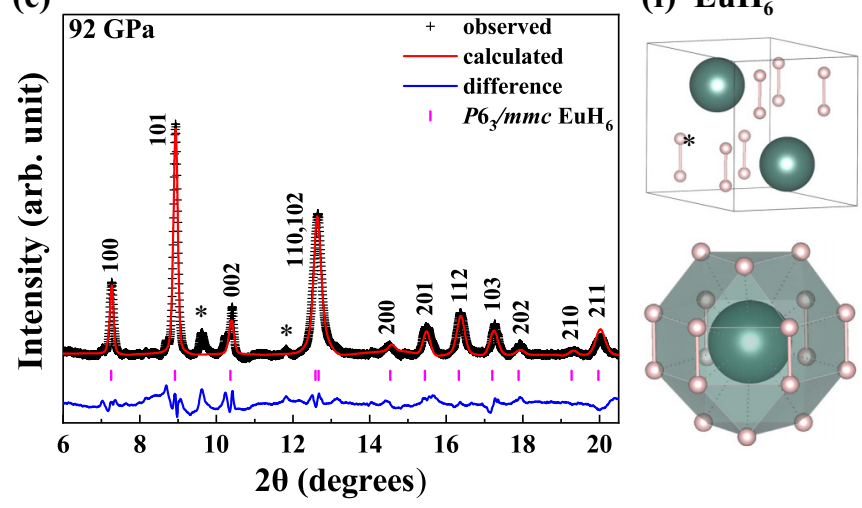

FIG. 1. Rietveld refinement of the experimental XRD pattern (left panel) and crystal structures (right panel) of (a), (b) $\mathrm{EuH}_{3}$ at $80 \mathrm{GPa}$, (c), (d) $\mathrm{EuH}_{5}$ at $130 \mathrm{GPa}$, and (e), (f) nonclathrate $\mathrm{EuH}_{6}$ at $92 \mathrm{GPa}$. Large and small balls represent the Eu atoms and $\mathrm{H}$ atoms, respectively.

structure, each Eu atom is surrounded by $24 \mathrm{H}$ atoms forming a $\mathrm{H}_{24}$ cage and each cage is composed of six squares and eight hexagons [Fig. 2(b)]. The H-H distance is $1.3 \AA$ and the nearest Eu-H distance is $2.051 \AA$ at $152 \mathrm{GPa}$. This structure was first predicted in $\mathrm{CaH}_{6}$ [5], but first synthesized in $\mathrm{YH}_{6}$ $[19,21]$. $\mathrm{EuH}_{6}$ is the second synthesized example and the first hexahydride synthesized in lanthanide hydrides. During the release of pressure, the clathrate $\mathrm{EuH}_{6}$ can be stabilized to at least $87 \mathrm{GPa}$, at a lower pressure which gradually decomposes into the $\mathrm{EuH}_{5}$ phase and $\mathrm{H}_{2}$ [Fig. 3(a)].

Sample C4 was heated to $2800 \mathrm{~K}$ with a loaded pressure at $170 \mathrm{GPa}$, aiming to synthesize superhydrides with a higher hydrogen content. The analysis of the measured XRD patterns 
(a)
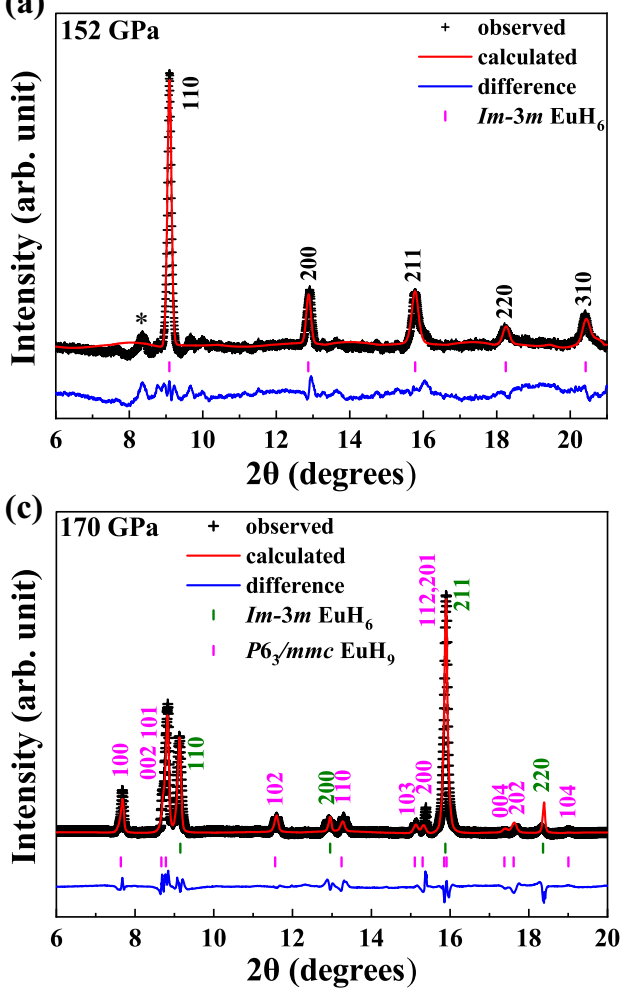

(d) $\mathrm{EuH}_{9}$

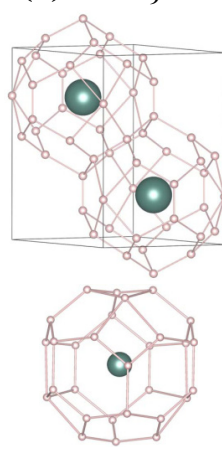

FIG. 2. Rietveld refinement of the experimental XRD pattern (left panel) and crystal structures (right panel) of (a), (b) clathrate $\mathrm{EuH}_{6}$ at $152 \mathrm{GPa}$, and (c), (d) the mixture of clathrate $\mathrm{EuH}_{6}$ and $\mathrm{EuH}_{9}$ at $170 \mathrm{GPa}$. Large and small balls represent the Eu atoms and $\mathrm{H}$ atoms, respectively.

showed that besides $\mathrm{EuH}_{6}$, the clathrate stoichiometry $\mathrm{EuH}_{9}$ predicted in our previous work [10] was successfully synthesized [Fig. 2(c)] at this condition. The weight fractions of the $\mathrm{EuH}_{6}$ and $\mathrm{EuH}_{9}$ phases in the mixture were calculated by Rietveld refinement and estimated to be $46.43 \%$ and $53.56 \%$, respectively. $\mathrm{EuH}_{9}$ was found to be isostructural to the earlier synthesized $\mathrm{ThH}_{9}$ [16], $\mathrm{YH}_{9}$ [20,21], $\mathrm{CeH}_{9}$ [14,15], $\mathrm{NdH}_{9}$ [18], and $\mathrm{PrH}_{9}$ [27] [Fig. 2(d)], in which each metal atom is surrounded by $29 \mathrm{H}$ atoms forming a $\mathrm{H}_{29}$ cage and each cage is composed of six irregular squares, six pentagons, and six hexagons. We note this phase has been reproduced by another work after we reported it in the initial version [56]. It should be noted that there are still some unidentified XRD patterns marked by asterisks, which may be related to the unexpected $\mathrm{Eu}_{x} \mathrm{H}_{y}$ compounds due to the temperature and pressure gradients in the laser-heated diamond-anvil cell experiments. In this research, we have carried out Raman measurements on the synthesized $\mathrm{EuH}_{x}$, but a Raman signal only for diamond and hydrogen could be detected.

The existence of $f$ electrons in lanthanide (except La) usually leads to the appearance of magnetism in the hydrides $[18,27]$. A $b$ initio calculations predicted that all neodymium superhydrides in Ref. [18] have antiferromagnetic order at high pressures. Given the local feature of seven $f$ electrons for the Eu element even up to $119 \mathrm{GPa}$, we have performed spin-polarized calculations on our synthesized Eu-H system. In order to investigate the magnetic configurations of these
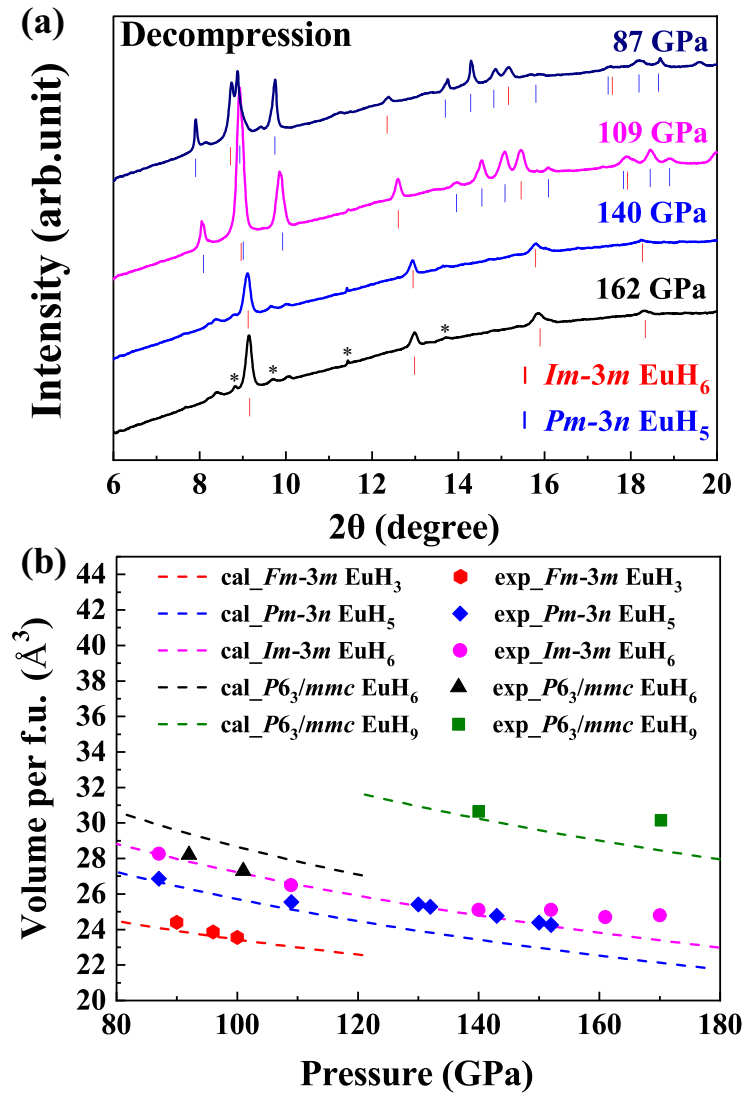

FIG. 3. (a) Experimental XRD patterns during decompression of sample $\mathrm{C} 3$ in the pressure range of 162-87 GPa. (b) The comparison of fitting EOS of all stoichiometries with the experimental $P-V$ data. The dashed curves represent the calculated EOS. The symbols represent the experimental result.

structures, we built up a supercell of four metal Eu atoms (Fig. S2) except for $\mathrm{EuH}_{5}$ by using the derivative structure enumeration library ENUMLIB [57], where all magnetic configurations were calculated with experimental lattice parameters. As a result, a strong magnetic moment was found in all the synthesized Eu-H compounds (Table S2) with $\sim 7 \mu_{B}$ per Eu atom at a pressure range of $80-180 \mathrm{GPa}$ (Fig. S3 [40]). Further simulations show the ferromagnetic feature of $P m \overline{3} n \mathrm{EuH}_{5}$ and $\operatorname{Im} \overline{3} m \mathrm{EuH}_{6}$, and the antiferromagnetic feature of $F m \overline{3} m$ $\mathrm{EuH}_{3}, P 6_{3} / m m c \mathrm{EuH}_{6}$, and $P 6_{3} / m m c \mathrm{EuH}_{9}$.

Furthermore, we fitted the EOS of all stoichiometries with the consideration of magnetism and spin-orbit coupling and compared with the experimental pressure-volume data [Fig. 3(b)]. It can be found that the experimental unit cell parameters and volumes are in good agreement with the theoretical data, which give further support to the validity of the structures and stoichiometries we identified from the XRD data.

To identify the bonding nature of hydrogen in clathrate superhydride, the electron localization function (ELF) was also calculated in $\mathrm{EuH}_{6}$ and $\mathrm{EuH}_{9}$ (Fig. S2 [40]). The weak electron localization $(0.5-0.6)$ between $\mathrm{H}$ atoms indicates weak covalent bond characteristics and a three-dimensional network structure in the $\mathrm{H}$ cages. An analysis of the electronic band structure illustrates that all the stoichiometries synthesized in 
this work present a metallic character at high pressures owing to the overlap of the conduction and valence bands at the Fermi level as shown in Figs. S4 and S5 [40].

Electrical resistance measurements as an efficient way to characterize the superconducting transition or magnetic order transition have been carried out using a four-probe technique for two samples after heating to about 1500 and $2000 \mathrm{~K}$ at $140 \mathrm{GPa}$, respectively, as shown in Fig. S7. The resistance decreases linearly during cooling at temperatures above $225 \mathrm{~K}$ in sample 1 and $258 \mathrm{~K}$ in sample 2, indicating their metallic nature. At lower temperatures, the slopes of the resistancetemperature $(R-T)$ curves become larger, as indicated by the red lines, implying an increase of the rates of resistance drop, which, however, are still very slow compared with the typical superconducting transition where resistance normally drops to zero within $10 \mathrm{~K}$. Interestingly, the profiles of the $R$ - $T$ curves are similar to the magnetic transition in lanthanide elements such as Eu [30] and Tb [58]. Referring to the synthesized conditions of different superhydrides in the above XRD measurements, we infer that the temperatures of 225 and $258 \mathrm{~K}$ at the kink points may indicate the Curie temperature of $P m \overline{3} n$ $\mathrm{EuH}_{5}$ and $\operatorname{Im} \overline{3} m \mathrm{EuH}_{6}$, respectively. A detailed study of the magnetic order transition in the Eu-H system is beyond the scope of this paper, but the current work may shed light on future experiments on magnetic superhydrides.

\section{CONCLUSION}

In summary, we have successfully synthesized a series of europium superhydrides, including $\mathrm{EuH}_{3}, \mathrm{EuH}_{5}, \mathrm{EuH}_{6}$, and $\mathrm{EuH}_{9}$, in the pressure range of $80-170 \mathrm{GPa}$. Among these hydrides, $\mathrm{EuH}_{6}$ and $\mathrm{EuH}_{9}$ have clathrate structures with an atomiclike hydrogen sublattice surrounding Eu atoms. Two nonclathrate structured phases of $\mathrm{EuH}_{5}$ and $\mathrm{EuH}_{6}$ are reported here for the prototype structure models in a system of lanthanide superhydrides. The calculation results show that all europium hydrides exhibit strong magnetism at the pressure range considered in the present study. This work paves the way for further experimental investigations on the complex mechanism of magnetism, the strongly correlated effect, and superconductivity on Eu superhydrides.

\section{ACKNOWLEDGMENTS}

This work was supported by the Major Program of the National Natural Science Foundation of China (Grant No. 52090024), Strategic Priority Research Program of Chinese Academy of Sciences (Grant No. XDB33000000), National Key R\&D Program of China (Grant No. 2018YFA0305900), National Natural Science Foundation of China (Grants No. 11874175, No. 12074139, No. 12074138, No. 11874176 , No. 12034009, and No. 11974134), Jilin Province Outstanding Young Talents Project (Grant No. 20190103040JH), and Program for JLU Science and Technology Innovative Research Team (JLUSTIRT). We used the computing facilities at the High-Performance Computing Centre of Jilin University and Tianhe2-JK at the Beijing Computational Science Research Centre. XRD measurement were performed at BL10XU/SPring-8, Shanghai Synchrotron Radiation Facility Beamline BL15U1 and Beijing Synchrotron Radiation Facility HP-Station $4 \mathrm{~W} 2$.

The authors declare no competing interests.
[1] N. W. Ashcroft, Metallic Hydrogen: A High-Temperature Superconductor? Phys. Rev. Lett. 21, 1748 (1968).

[2] P. Loubeyre, F. Occelli, and P. Dumas, Synchrotron infrared spectroscopic evidence of the probable transition to metal hydrogen, Nature (London) 577, 631 (2020).

[3] M. I. Eremets, A. P. Drozdov, P. P. Kong, and H. Wang, Semimetallic molecular hydrogen at pressure above $350 \mathrm{GPa}$, Nat. Phys. 15, 1246 (2019).

[4] P. Dalladay-Simpson, R. T. Howie, and E. Gregoryanz, Evidence for a new phase of dense hydrogen above 325 gigapascals, Nature (London) 529, 63 (2016).

[5] H. Wang, J. S. Tse, K. Tanaka, T. Iitaka, and Y. Ma, Superconductive sodalite-like clathrate calcium hydride at high pressures, Proc. Natl. Acad. Sci. USA 109, 6463 (2012).

[6] H. W. Kroto, J. R. Heath, S. C. O'Brien, R. F. Curl, and R. E. Smalley, $\mathrm{C}_{60}$ : buckminsterfullerene, Nature (London) 318, 162 (1985)

[7] J. S. Kasper, P. Hagenmuller, M. Pouchard, and C. Cros, Clathrate structure of silicon $\mathrm{Na}_{8} \mathrm{Si}_{46}$ and $\mathrm{Na}_{x} \mathrm{Si}_{136}(x<11)$, Science 150, 1713 (1965).

[8] Y. Li, J. Hao, H. Liu, J. S. Tse, Y. Wang, and Y. Ma, Pressurestabilized superconductive yttrium hydrides, Sci. Rep. 5, 9948 (2015).

[9] X. Feng, J. Zhang, G. Gao, H. Liu, and H. Wang, Compressed sodalite-like $\mathrm{MgH}_{6}$ as a potential high-temperature superconductor, RSC Adv. 5, 59292 (2015).
[10] F. Peng, Y. Sun, C. J. Pickard, R. J. Needs, Q. Wu, and Y. Ma, Hydrogen Clathrate Structures in Rare Earth Hydrides at High Pressures: Possible Route to Room-Temperature Superconductivity, Phys. Rev. Lett. 119, 107001 (2017).

[11] H. Liu, I. I. Naumov, R. Hoffmann, N. W. Ashcroft, and R. J. Hemley, Potential high- $T_{c}$ superconducting lanthanum and yttrium hydrides at high pressure, Proc. Natl. Acad. Sci. USA 114, 6990 (2017).

[12] M. Somayazulu, M. Ahart, A. K. Mishra, Z. M. Geballe, M. Baldini, Y. Meng, V. V. Struzhkin, and R. J. Hemley, Evidence for Superconductivity above $260 \mathrm{~K}$ in Lanthanum Superhydride at Megabar Pressures, Phys. Rev. Lett. 122, 027001 (2019).

[13] A. P. Drozdov, P. Kong, V. S. Minkov, S. P. Besedin, M. A. Kuzovnikov, S. Mozaffari, L. Balicas, F. F. Balakirev, D. E. Graf, V. B. Prakapenka et al., Superconductivity at $250 \mathrm{~K}$ in lanthanum hydride under high pressures, Nature (London) 569, 528 (2019)

[14] X. Li, X. Huang, D. Duan, C. J. Pickard, D. Zhou, H. Xie, Q. Zhang, Y. Huang, Q. Zhou, B. Liu et al., Polyhydride $\mathrm{CeH}_{9}$ with an atomic-like hydrogen clathrate structure, Nat. Commun. 10, 3461 (2019).

[15] N. P. Salke, M. M. Esfahani, Y. Zhang, I. A. Kruglov, J. Zhou, Y. Wang, E. Greenberg, V. B. Prakapenka, J. Liu, A. R. Oganov et al., Synthesis of clathrate cerium superhydride $\mathrm{CeH}_{9}$ at 80 $100 \mathrm{GPa}$ with atomic hydrogen sublattice, Nat. Commun. 10, 4453 (2019). 
[16] D. V. Semenok, A. G. Kvashnin, A. G. Ivanova, V. Svitlyk, V. Y. Fominski, A. V. Sadakov, O. A. Sobolevskiy, V. M. Pudalov, I. A. Troyan, and A. R. Oganov, Superconductivity at $161 \mathrm{~K}$ in thorium hydride $\mathrm{ThH}_{10}$ : Synthesis and properties, Mater. Today 33, 36 (2020).

[17] T. Matsuoka, M. Hishida, K. Kuno, N. Hirao, Y. Ohishi, S. Sasaki, K. Takahama, and K. Shimizu, Superconductivity of platinum hydride, Phys. Rev. B 99, 144511 (2019).

[18] D. Zhou, D. V. Semenok, H. Xie, X. Huang, D. Duan, A. Aperis, P. M. Oppeneer, M. Galasso, A. I. Kartsev, A. G. Kvashnin et al., High-pressure synthesis of magnetic neodymium polyhydrides, J. Am. Chem. Soc. 142, 2803 (2020).

[19] I. A. Troyan, D. V. Semenok, A. G. Kvashnin, A. V. Sadakov, O. A. Sobolevskiy, V. M. Pudalov, A. G. Ivanova, V. B. Prakapenka, E. Greenberg, A. G. Gavriliuk et al., Anomalous high-temperature superconductivity in $\mathrm{YH}_{6}, \mathrm{Adv}$. Mater. 33, 2006832 (2021).

[20] E. Snider, N. Dasenbrock-Gammon, R. McBride, X. Wang, N. Meyers, K. V. Lawler, E. Zurek, A. Salamat, and R. P. Dias, Synthesis of Yttrium Superhydride Superconductor with a Transition Temperature up to $262 \mathrm{~K}$ by Catalytic Hydrogenation at High Pressures, Phys. Rev. Lett. 126, 117003 (2021).

[21] P. Kong, V. S. Minkov, M. A. Kuzovnikov, A. P. Drozdov, S. P. Besedin, S. Mozaffari, L. Balicas, F. F. Balakirev, V. B. Prakapenka, S. Chariton et al., Superconductivity up to $243 \mathrm{~K}$ in the yttrium-hydrogen system under high pressure, Nat. Commun. 12, 5075 (2021).

[22] D. V. Semenok, I. A. Troyan, A. G. Ivanova, A. G. Kvashnin, I. A. Kruglov, M. Hanfland, A. V. Sadakov, O. A. Sobolevskiy, K. S. Pervakov, I. S. Lyubutin et al., Superconductivity at $253 \mathrm{~K}$ in lanthanum-yttrium ternary hydrides, Materials Today 48,18 (2021).

[23] W. Chen, D. V. Semenok, A. G. Kvashnin, X. Huang, I. A. Kruglov, M. Galasso, H. Song, D. Duan, A. F. Goncharov, V. B. Prakapenka et al., Synthesis of molecular metallic barium superhydride: pseudocubic $\mathrm{BaH}_{12}$, Nat. Commun. 12, 273 (2021).

[24] L. Ma, K. Wang, Y. Xie, X. Yang, Y. Wang, M. Zhou, H. Liu, X. Yu, Y. Zhao, H. Wang et al., Experimental observation of superconductivity at $215 \mathrm{~K}$ in calcium superhydride under high pressures, arXiv:2103.16282.

[25] Z. W. Li, X. He, C. L. Zhang, S. J. Zhang, S. M. Feng, X. C. Wang, R. C. Yu, and C. Q. Jin, Superconductivity above $200 \mathrm{~K}$ observed in superhydrides of calcium, arXiv:2103.16917.

[26] W. Chen, D. V. Semenok, X. Huang, H. Shu, X. Li, D. Duan, T. Cui, and A. R. Oganov, High-Temperature Superconducting Phases in Cerium Superhydride with a $T_{c}$ up to $115 \mathrm{~K}$ below a Pressure of 1 Megabar, Phys. Rev. Lett. 127, 117001 (2021).

[27] D. Zhou, D. V. Semenok, D. Duan, H. Xie, W. Chen, X. Huang, X. Li, B. Liu, A. R. Oganov, and T. Cui, Superconducting praseodymium superhydrides, Sci. Adv. 6, eaax6849 (2020).

[28] J. C. Bunzli, Europium in the limelight, Nat. Chem. 2, 696 (2010).

[29] T. Wang, D. Zhao, X. Guo, J. Correa, B. L. Riehl, and W. R. Heineman, Carbon nanotube-loaded Nafion film electrochemical sensor for metal ions: Europium, Anal. Chem. 86, 4354 (2014).

[30] M. Debessai, T. Matsuoka, J. J. Hamlin, J. S. Schilling, and K. Shimizu, Pressure-Induced Superconducting State of Europium Metal at Low Temperatures, Phys. Rev. Lett. 102, 197002 (2009).
[31] B. Johansson and A. Rosengren, Generalized phase diagram for the rare-earth elements: Calculations and correlations of bulk properties, Phys. Rev. B 11, 2836 (1975).

[32] A. Rosengren and B. Johansson, Alloy theory of the intermediate valence state: Application to europium metal, Phys. Rev. B 13, 1468 (1976).

[33] W. Bi, J. Lim, G. Fabbris, J. Zhao, D. Haskel, E. E. Alp, M. Y. Hu, P. Chow, Y. Xiao, W. Xu et al., Magnetism of europium under extreme pressures, Phys. Rev. B 93, 184424 (2016).

[34] H. Saitoh, A. Machida, T. Matsuoka, and K. Aoki, Phase diagram of the Eu-H system at high temperatures and high hydrogen pressures, Solid State Commun. 205, 24 (2015).

[35] T. Matsuoka et al., Structural and Valence Changes of Europium Hydride Induced by Application of High-Pressure $\mathrm{H}_{2}$, Phys. Rev. Lett. 107, 025501 (2011).

[36] Y. Akahama and H. Kawamura, Pressure calibration of diamond anvil Raman gauge to $310 \mathrm{GPa}$, J. Appl. Phys. 100, 043516 (2006).

[37] N. Hirao, S. I. Kawaguchi, K. Hirose, K. Shimizu, E. Ohtani, and Y. Ohishi, New developments in high-pressure X-ray diffraction beamline for diamond anvil cell at SPring-8, Matter Radiat. Extremes. 5, 018403 (2020).

[38] C. Prescher and V. B. Prakapenka, DIOPTAS: a program for reduction of two-dimensional X-ray diffraction data and data exploration, High Pressure Res. 35, 223 (2015).

[39] B. H. Toby, EXPGUI, a graphical user interface for GSAS, J. Appl. Crystallogr. 34, 210 (2001).

[40] See Supplemental Material at http://link.aps.org/supplemental/ 10.1103/PhysRevResearch.3.043107 for full lists of detailed crystal information, magnetic configurations, and calculated electronic band and electrical resistance measurements.

[41] P. Hohenberg and W. Kohn, Inhomogeneous electron gas, Phys. Rev. 136, B864 (1964).

[42] W. Kohn and L. J. Sham, Self-consistent equations including exchange and correlation effects, Phys. Rev. 140, A1133 (1965).

[43] J. P. Perdew, K. Burke, and M. Ernzerhof, Generalized Gradient Approximation Made Simple, Phys. Rev. Lett. 77, 3865 (1996).

[44] P. E. Blöchl, Projector augmented-wave method, Phys. Rev. B 50, 17953 (1994).

[45] G. Kresse and D. Joubert, From ultrasoft pseudopotentials to the projector augmented-wave method, Phys. Rev. B 59, 1758 (1999).

[46] G. Kresse and J. Hafner, Ab initio molecular dynamics for openshell transition metals, Phys. Rev. B 48, 13115 (1993).

[47] G. Kresse and J. Hafner, Ab initio molecular-dynamics simulation of the liquid-metal-amorphous-semiconductor transition in germanium, Phys. Rev. B 49, 14251 (1994).

[48] G. Kresse and J. Furthmiller, Efficiency of ab-initio total energy calculations for metals and semiconductors using a plane-wave basis set, Comput. Mater. Sci. 6, 15 (1996).

[49] R. J. Husband, I. Loa, G. W. Stinton, S. R. Evans, G. J. Ackland, and M. I. McMahon, Europium-IV: An Incommensurately Modulated Crystal Structure in the Lanthanides, Phys. Rev. Lett. 109, 095503 (2012).

[50] F. Birch, Finite elastic strain of cubic crystals, Phys. Rev. 71, 809 (1947).

[51] A. J. Maeland and D. E. Holmes, Inelastic neutron scattering spectra from lanthanum dihydride and lanthanum trihydride, J. Chem. Phys. 54, 3979 (1971). 
[52] R. E. Rundle, The hydrogen positions in uranium hydride by neutron diffraction, J. Am. Chem. Soc. 73, 4172 (1951).

[53] Y. Wang, J. Lv, L. Zhu, and Y. Ma, CALYPSO: A method for crystal structure prediction, Comput. Phys. Commun. 183, 2063 (2012).

[54] Y. Wang, J. Lv, L. Zhu, and Y. Ma, Crystal structure prediction via particle-swarm optimization, Phys. Rev. B 82, 094116 (2010).

[55] S. Qian, X. Sheng, X. Yan, Y. Chen, and B. Song, Theoretical study of stability and superconductivity of $\mathrm{ScH}_{n}(n=4-8)$ at high pressure, Phys. Rev. B 96, 094513 (2017).
[56] D. V. Semenok, D. Zhou, A. G. Kvashnin, X. Huang, M. Galasso, I. A. Kruglov, A. G. Ivanova, A. G. Gavriliuk, W. Chen, N. V. Tkachenko et al., Novel strongly correlated europium superhydrides, J. Phys. Chem. Lett. 12, 32 (2020).

[57] G. L. W. Hart, L. J. Nelson, and R. W. Forcade, Generating derivative structures at a fixed concentration, Comput. Mater. Sci. 59, 101 (2012).

[58] J. Lim, G. Fabbris, D. Haskel, and J. S. Schilling, Anomalous pressure dependence of magnetic ordering temperature in $\mathrm{Tb}$ revealed by resistivity measurements to $141 \mathrm{GPa}$ : Comparison with Gd and Dy, Phys. Rev. B 91, 174428 (2015). 